\title{
Collection Efficiency Enhancement of Cyclone Filtration System
}

\author{
A. H. A. Hamid, L. H. Kamaruhen, M. H. M. Noh
}

\begin{abstract}
The present study focuses on improving the cyclone abatement device that is used in the agriculture and processing industries such as palm oil mill industry. The air flow trajectories are simulated and analyzed using the Computational Fluid Dynamics (CFD). Cyclones use the principle of inertia to remove particulate matter from flue gases. The current design of the cyclone is not able to collect particulate matter that is smaller than 10 micrometers effectively. This will result in negative effect to the environment as well as to the community. Thus, it is essential to modify the cyclone filters and study its effects on the overall performance of the cyclones. The principle idea of the geometrical modification is to alter the boundary layer adjacent to the inner wall of the cyclone in order to enhance the cyclone performance. The collection efficiency and pressure drop are the main factors that can affect the performance of the cyclone. The separator plates are designed in certain sizes to be placed inside the cyclone with different positions. It is shown that the position of the plate inside the cyclone significantly affects the performance of the cyclone filter. The cyclone with $145 \mathrm{~mm}$ separator plate has shown to produce highest efficiency marked $45.23 \%$ relative to the standard cyclone. In overall, the separator plate significantly increases the cyclone performance.
\end{abstract}

Index Terms: separator plate; air flow trajectories; cyclone; collection efficiency; particulate matter

\section{INTRODUCTION}

Cyclone separators or centrifugal separators are one of the types of air pollution devices. These devices are also called as "the pre-cleaners" as they are used to remove larger pieces of particulate matter before it emits to the atmosphere. These devices are relatively easy to manufacture due to the fact that it contains no moving parts, hence, less maintenance problems and adaptable to the harsh conditions. Due to these advantages, the cyclone separators have been widely used as particle removal devices in engineering and scientific fields. The fundamental working principle is rather simple: the

Manuscript published on November 30, 2019

* Correspondence Author

A. H. A. Hamid*, Faculty of Mechanical Engineering, Universiti Teknologi MARA, 40450, Shah Alam, Selangor, Malaysia

L. H. Kamaruhen, Faculty of Mechanical Engineering, Universiti Teknologi MARA, 40450, Shah Alam, Selangor, Malaysia,

M. H. M. Noh, Faculty of Mechanical Engineering, Universiti Teknologi MARA, 40450, Shah Alam, Selangor, Malaysia

(C) The Authors. Published by Blue Eyes Intelligence Engineering and Sciences Publication (BEIESP). This is an open access article under the CC-BY-NC-ND

license (http://creativecommons.org/licenses/by-nc-nd/4.0/) swirling motion inside of the separators produces the centrifugal force in the solid particles, which drives the separation process [1].

First, particle laden air stream enters tangentially at the top of the barrel and swirling downward into the cone. The motion forms an outer vortex and pushes the particles towards the outer wall. The particles with higher densities spiral in the downward direction, which are then collected in the particle collector bin attached to the bottom outlet of the cyclone separator [2].

When the flow reaches the bottom part of the cone, it swirls upward, forming an inner vortex with vertically opposite directions [3]. This double vortex phenomenon creates a strong turbulence flow that is used to separate particles from the air or phases with different densities.

The filtered air flows through the outlet, located on the top of cyclone separator. However, the turbulence within the separators disperses the particles and thus increases the probabilities that the particles are caught in the exit stream. This will definitely reduce the capture efficiency and thus the overall performance of the separators. It is known that there are various geometrical and operational parameters that influence the cyclone performance. In the present investigation, a simple geometrical device, called separator plate, is retrofitted in the typical cyclone separator in order to minimize the numbers of particles released along with the filtered air.

\section{METHODOLOGY}

\section{A. Geometry of Cyclone}

The geometry of the cyclone was designed using CAD software on a pilot scale to perform the optimization studies and reduce computation time. The geometrical dimensions of the cyclone are summarized in TableI and the schematic representation is shown in Fig. 1.

Table-I: Geometrical dimensions of cyclone

\begin{tabular}{lcc} 
Parameters & $\begin{array}{c}\text { Abbreviation } \\
\text { Cyclone diameter }\end{array}$ & $\begin{array}{c}\text { Dimensions } \\
\text { (mm) }\end{array}$ \\
\hline Inlet height & $\mathrm{D}$ & 290 \\
Inlet width & $\mathrm{A}$ & 145 \\
Cone-tip diameter & $\mathrm{B}$ & 58 \\
Exit pipe diameter & $\mathrm{D}_{\mathrm{c}}$ & 107.3 \\
Cylindrical part height & $\mathrm{D}_{\mathrm{x}}$ & 116 \\
\hline
\end{tabular}




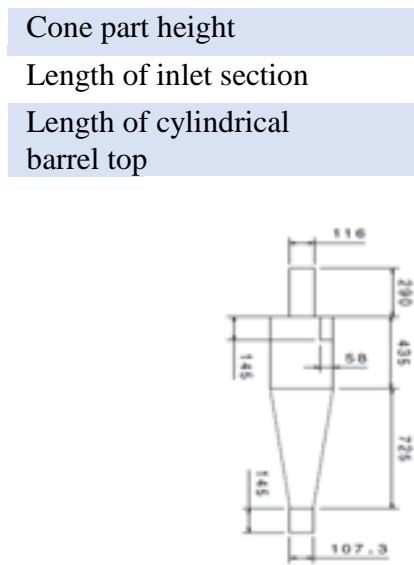

(a)

$\begin{array}{cc}\mathrm{h}_{\mathrm{c}} & 725 \\ \mathrm{~L}_{\mathrm{i}} & 400.2 \\ \mathrm{~L} & 290\end{array}$

\section{0}

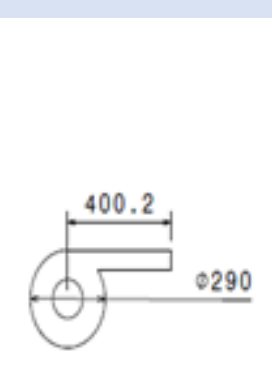

(b)

Fig. 1. Geometry of base case cyclone (without separator plate) (a) side view and (b) top view.

\section{B. Separator Plate}

The present study simulated three types of geometrical dimensions (Geometries A, B and C) of the cyclone separator. The separator plate is embedded within the cylindrical barrel of the cyclone in order to investigate their effects on the effect of the cyclone's performance. TableII shows the comparison between the different designs.

Table-II: Geometrical configurations of the investigated cyclone filters

\begin{tabular}{|c|l|l|}
\hline Geometry & $\begin{array}{l}\text { Diameter of } \\
\text { Separator Plate }\end{array}$ & $\begin{array}{l}\text { Position of Separator } \\
\text { Plate }\end{array}$ \\
\hline A & No plate added & No plate added \\
\hline B1 & \multirow{4}{*}{$145 \mathrm{~mm}$} & Bottom \\
\cline { 1 - 1 } B2 & & Middle \\
\cline { 1 - 1 } & \multirow{4}{*}{$72.5 \mathrm{~mm}$} & Top \\
\hline B1 & Bottom \\
\hline C2 & Middle \\
\hline C3 & & Top \\
\hline
\end{tabular}

Geometry A is a base case geometry (cyclone filter without separator plate, as shown in Fig. 1). Geometry B is a cyclone separator with $145 \mathrm{~mm}$ diameter of separator plate (Fig. 3), as depicted in Fig. 4. Geometry C (Fig. 5) is a cyclone separator with $72.5 \mathrm{~mm}$ diameter of separator plate, as shown in Fig. 2 The cyclone with separator plates were tested with different positions which are top, middle and bottom. The position is measured from the top of the cyclone design. The study was conducted based on CFD using the finite volume method.

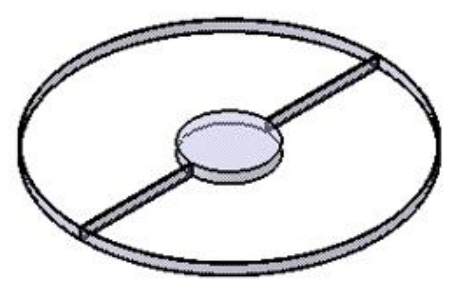

Fig. 2. Separator plate with diameter of $72.5 \mathrm{~mm}$

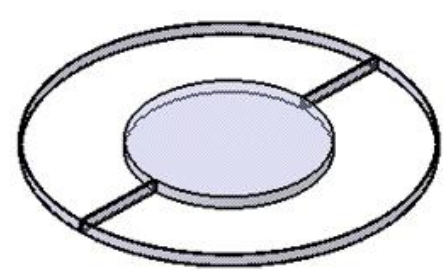

Fig. 3. Separator plate with diameter of $\mathbf{1 4 5} \mathbf{~ m m}$

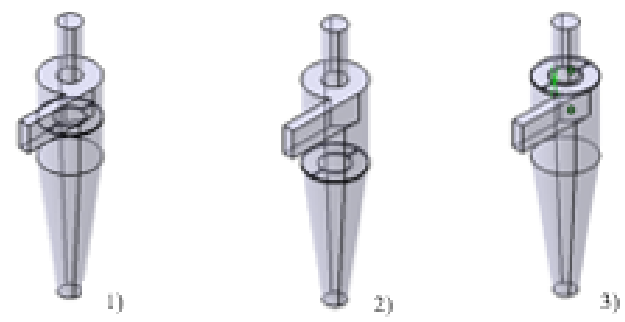

Fig. 4. Geometry B 1) middle 2) bottom 3) top
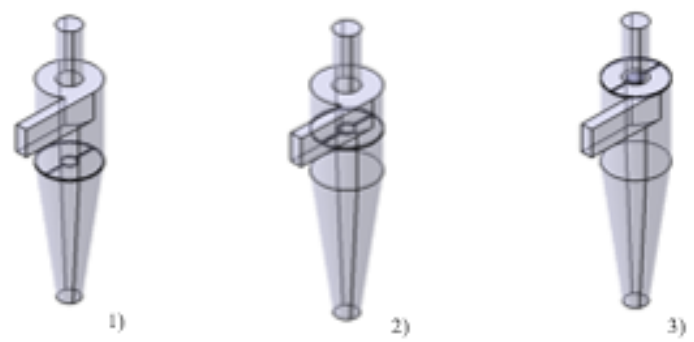

Fig. 5. Geometry C 1) bottom 2) middle 3) top

\section{Simulation Setup}

The simulation of the cyclone separators was conducted using tetrahedral meshing for complex geometry. The simulation was conducted based on Reynolds Averaged Navier Stokes (RANS) as the turbulent approach [4]. The type of RANS used is 3D unstable Reynolds Stresses Model (RSM) due to the powerful turbulence flow inside the cyclone separator. It was therefore crucial to simulate the flow's turbulence behavior correctly. The advancement of computational technology has allowed the use of several turbulence models to predict the flow field. Starting variety from normal k-epsilon to multiphase model such as Langrangian Multiphase Model (LMP) was adopted. In the cyclone separator model, the normal k-epsilon and RNG k-epsilon models are not completely optimized for heavily flowing flows. RSM is more capable to predict the complicated flow.

The standard wall function was used to solve turbulent flow problems in the wall regions. The fluid phase of particulates was simulated as dispersion model. Discrete Phase Model (DPM) with one way coupling was used for the description of the dispersed phase [5]. The diameter of particle injected in the cyclone was $2.5 \mu \mathrm{m}$ with the density of $2770 \mathrm{~kg} / \mathrm{m}^{3}$.

The equation of particle motion is based on the assumptions that the particles are perfectly spherical in shape [6]. 
Furthermore, it is assumed that the volume fraction of the dispersed phase in the continuous region is negligible, hence, no influence was due to the presence of the particles [7]. Additionally, it is assumed that there are zero inter-particle interactions since the flow is continuous and thus the particle is diluted. [8]. Finally, collisions between particles and the wall are assumed to be perfectly elastic. Under these assumptions, the particle trajectories are obtained by integrating the force balance on the particle [9]. The fractional efficiency was calculated based on equation (1).

$$
\text { Efficiency }=\frac{\text { trapped }}{\text { number of tracked-incomplete }}
$$

The "tracked" is the number of particle tracks that is injected into the domain. The "trapped" is defined as the total number of particles that is successfully collected by the cyclone separator, while the "incomplete" refers to the number of particles that stay in the cyclone without being collected nor emits. With regard to the convergence criteria, there are two aspects that are considered, i.e. the scaled residuals (the threshold is set at $1 \times 10^{-5}$ ) and some representative quantities such as velocity and pressure (until they have reached statistically steady state [10]). Although the present simulations were conversed at about $(\mathrm{t}=1.5-1.6 \mathrm{~s})$, they were only terminated at $\mathrm{t}=2 \mathrm{~s}$ to get more accurate time averaged values. All simulations have been converged with the selected time step of $1 \times 10^{-4} \mathrm{~s}$ [11].

\section{RESULT AND DISCUSSION}

In the present simulations, particle size $2.5 \mu \mathrm{m}$ was injected into the domain tangentially and the collection efficiency was measured for each case. The collection efficiency is normalized with respect to the base case without the separator plate (i.e. Geometry A with collection efficiency of 2.44\%). This low collection efficiency of Geometry A indicates a relatively amount of particle has been escaped to the environment.

The cyclone filter is modified by retrofitting a separator into the center of the filter. The separator is expected to somehow impose a resistance to the swirling flow at the core of the cyclone filter (as indicated by the yellow and red streamlines in Fig. 6), thus increasing the number of particles trapped inside the filter.

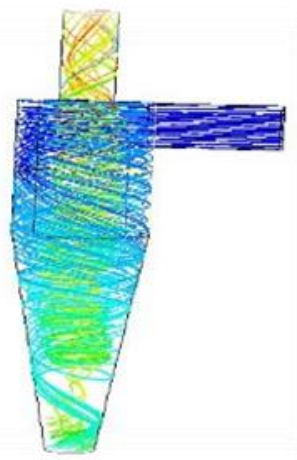

Fig. 6. Streamlines of tracked particles in the cyclone filter

The performance of cyclone separator with different diameters of separator plate (i.e. $72.5 \mathrm{~mm}$ and $145 \mathrm{~mm}$ for Geometry C and B, respectively) is shown in Fig. 7 for three different locations of bottom, middle and top. The addition of the separator plate in the cyclone separator has shown to significantly increase the number of particles trapped in the cyclone. The bigger the particle size, the quicker it moves to the cyclone's bottom. Smaller particles are more difficult to move under the separator plate. If the particle drops below the separator plate, it no longer passes through the centre of the cyclone.

Fig. 7 also indicates that cyclone with geometry type B (cyclone separator with $145 \mathrm{~mm}$ plate in middle position) showed the greatest effectiveness compared to other configurations. The collection efficiency increases by almost a factor of 19 relative to the case without the separator. This implies that the incorporation of the separator plate within the cyclone filter can boost the effectiveness of particulate trapping. Geometry B, however, showed a normalized collection efficiency of approximately 12, 37\% less than that of Geometry C. Less collection efficiency could give negative impact to the environment and also can affect the human respiratory system.

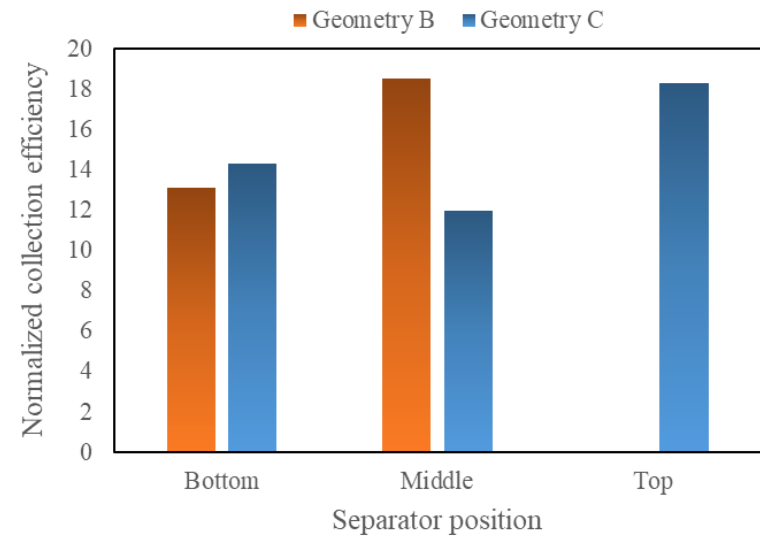

Fig. 7. Non-dimensionalized collection efficiency of cyclone separator with different sizes and positions

It is interesting to note that, when the separator plate is positioned at the bottom of the filter, the size of the separator plate plays a lesser role in the collection efficiency, as indicated in Fig. 7. The collection efficiency of both Geometries B and C are $13.1 \%$ and $14.3 \%$, respectively.

For the case where the separator is positioned at the top of the filter, Geometry B did not have an important impact on the cyclone's effectiveness since the plate itself blocked the flow of air out of the cyclone. Geometry C, however, showed a significant increase in the performance, where the collection efficiency is approximately equal to that of Geometry B positioned at the mid of cyclone filter.

Another important parameter in the study of cyclone performance is pressure losses, which can have a significant impact on the overall performance of the cyclone and the required energy to mobilize the air. Pressure losses has been measured through the area-averaged pressures at the inlet and outlet sections. Comparison of cyclone pressure loss separator was performed on type A, B, and C geometry.

Blue Eyes Intelligence Engineering DOI:10.35940/ijrteD5195.118419

Journal Website: www.ijrte.org

\& Sciences Publication 
The pressure loss of flow within the cyclone filter for different diameterS and location of separator plate is shown in Fig. 8.

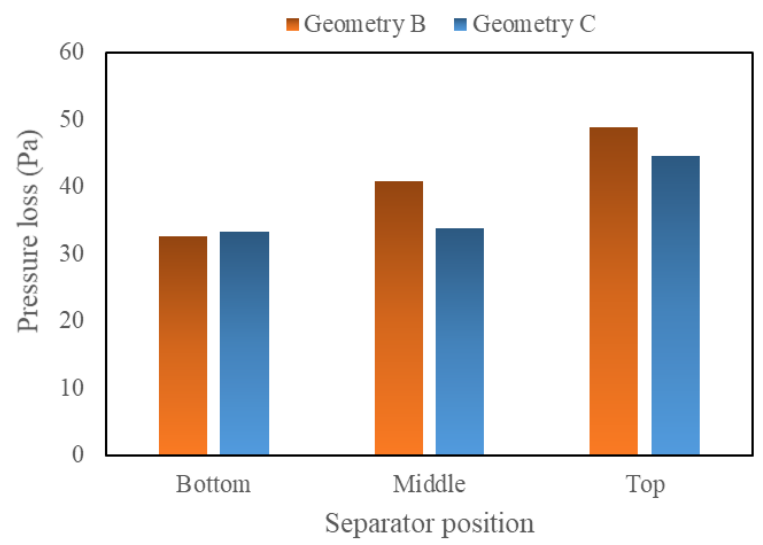

Fig. 8. Pressure loss $(\mathrm{Pa})$ plotted against separator position for two different separator plate diameters

It is observed that larger separator plate tends to increase the pressure loss within the cyclone filter. This observation is attributed to the fact that larger separator plate imposes a higher resistance towards the swirling air flows within the filter, thus contributing to a larger pressure drop. It is also noted that the trend of pressure loss is similar to that of collection efficiency. The plot of collection efficiency plotted against pressure loss (as shown in Fig. 9) revealed a proportionate trend between the two.

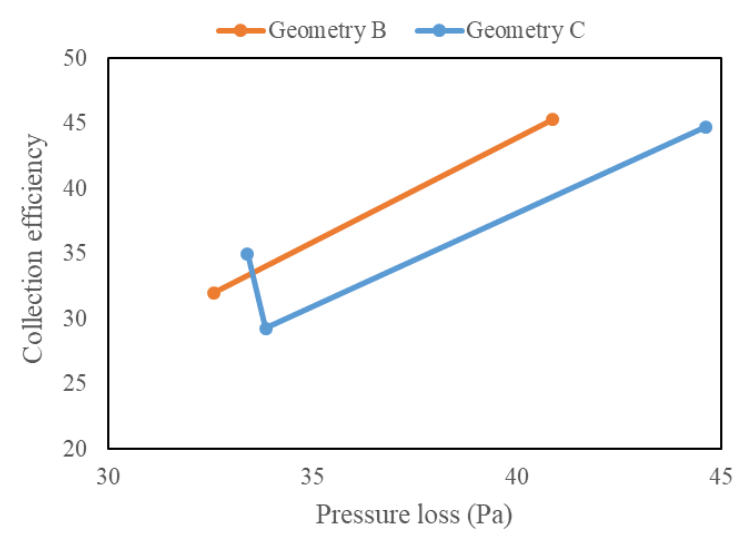

Fig. 9. Collection efficiency vs pressure loss

In general, when the pressure loss value is greater, the efficiency of the cyclone increases. The latter effect is desirable while the former is not. The two competing effects deserves further attentions in optimizing the design of the separator plate.

\section{CONCLUSION}

The impacts of the separator plate diameter and position on the general effectiveness of particulate matter collection and flow losses have been explored numerically.

It is shown that adding the separator plate in the abatement device increases the number of dusts trapped and thus the overall performance of the cyclone. Hence, it is anticipated that the addition of a separator plate in the cyclone separator enhances the quality of air emissions to the environment. The findings also suggest the potential of design optimization due to the competing effects of gain in both pressure loss and collection efficiency.

\section{REFERENCES}

1. J. Galins, A. Laizans, and A. Galins, "Increasing cyclone efficiency by using a separator plate," Rural Environ. Eng. Landsc. Archit., vol. 1, pp. 207-210, 2018.

2. S. Akhbarifar, M. Shirvani, S. Zahedi, M. R. Zahiri, and Y. Shamsaii, "Improving cyclone efficiency by recycle and jet impingement streams," Iran. J. Chem. Chem. Eng., vol. 30, no. 2, pp. 119-124, 2011.

3. L. Wang-li, "Theoretical sutdy of cyclone design," Texas A\&M University, 2003.

4. A. J. Hoekstra, J. J. Derksen, and H. E. A. Van Den Akker, "A CFD study on the performance of a high-efficiency gas cyclone," Am. Soc. Mech. Eng. Press. Vessel. Pip. Div. PVP, vol. 397 II, pp. 219-226, 1999.

5. S. Ganegama Bogodage and A. Y. T. Leung, "CFD simulation of cyclone separators to reduce air pollution," Powder Technol., vol. 286, pp. 488-506, 2015.

6. A.J. Hoekstra, "Gas Flow Field and Collection Efficiency of Cyclone Separators," Delft University of Technology, 2000.

7. S. S. Gawali and M. B. Bhambere, "Effect of Design and Operational Parameters on the Performance of Cyclone," Int. J. Mech. Eng. Robot. Res., vol. 4, no. 1, pp. 244-248, 2015.

8. K. Elsayed and C. Lacor, "The effect of vortex finder diameter on cyclone separator performance and flow field," in V European Conference on Computational Fluid Dynamics, 2010, pp. 14-17.

9. B. Zhao, H. Shen, and Y. Kang, "Development of a symmetrical spiral inlet to improve cyclone separator performance," Powder Technol., vol. 145, no. 1, pp. 47-50, 2004.

10. L. Wang, C. B. Parnell, Jr., B. W. Shaw, and R. E Lacey, "Analysis of Cyclone Collection Efficiency," Analysis of Cyclone Collection Efficiency, Nevada, Art no. 034114, 2003.

11. G. B. Sakura and A. Y. T. Leung, "Experimental Study of Particle Collection Efficiency of Cylindrical Inlet Type Cyclone Separator," Int. J. Environ. Sci. Dev., vol. 6, no. 3, pp. 160-164, 2015.

\section{AUTHORS PROFILE}

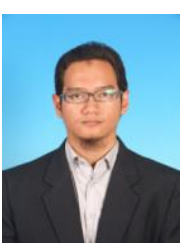

A. H. A. Hamid is an academic staff in the Faculty of Mechanical Engineering, Universiti Teknologi MARA, Malaysia. A. H. A. Hamid received his M.Sc. degree in 2008 from the same university. In 2016, A. H. A. Hamid graduated his PhD in Mechanical Engineering from Monash University, Melbourne, Australia. His fields of specialization are magnetohydrodynamics and heat transfer. His research interests include wake flow of a bluff body, magnetohydrodynamic flow, heat transfer, liquid atomization and renewable energy.

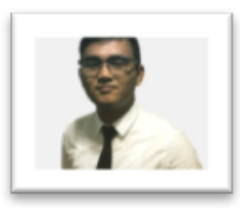

L. H. Kamaruhen currently is a final year student pursuing Bachelor Degree in Mechanical Engineering in Universiti Teknologi MARA (UiTM). He involved in designing handling system for UiTM Ecophoton solar racing team.

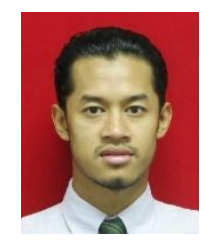

M. H. M. Noh is an academic staff in the Faculty of Mechanical Engineering, Universiti Teknologi MARA, Malaysia. M. H. M. Noh received his PhD from Nagoya University, Japan in the field of turbomachinery. His research interests include aerodynamics and gas turbine technologies. 